\title{
Influência da função distribuição de quebra na modelagem e simulação do circuito de moagem de Carajás - Parte 2 - Simulação
}

\author{
Influence of the distribution function on modeling and simulation \\ of the Carajás grinding circuit - Part 2 - simulation
}

\section{Bianca Foggiatto}

Mestranda em Engenharia Mineral

Escola Politécnica da USP

E-mail: bianca.foggiatto@gmail.com

\section{Homero Delboni Júnior}

Professor, Doutor do Departamento de Engenharia de Minas e de Petróleo

Escola Politécnica da USP

E-mail: hdelboni@usp.br

\section{Resumo}

Empregando-se o método descrito na Parte 1 desse artigo, foram realizadas caracterizações quanto à fragmentação dos principais tipos de minério de Carajás, provenientes das frentes de lavra das minas N4 e N5. A partir das características de tais minérios, foi empregado o modelo calibrado, para analisar, através de simulações, o desempenho do circuito de moagem de Carajás. Como resultado, foi possível verificar as variações de distribuição granulométrica do produto da moagem e do overflow dos ciclones simulados, bem como a carga circulante. As simulações conduzidas apontaram potenciais de processamento dos minérios cujo comportamento na moagem era até então desconhecido.

Palavras-chave: Moagem, modelagem, minério de ferro.

\section{Abstract}

Using the previously described method for appearance function determination, described in Part 1 of this article, the breakage characterization of the main Carajás ore types was carried out. Based on such characteristics, the ball mill circuit performance was evaluated through simulations. The model described in the first part was used. The results were assessed by comparing ball mill products and cyclone overflow size distribution, as well as simulated recirculating loads. The simulations indicated the potential for processing such ore types at the Carajas grinding circuit, which until now was unknown.

Keywords: Grinding, modeling, iron ore. 
Influência da função distribuição de quebra na modelagem e simulação do circuito de moagem de Carajás...

\section{Introdução}

Com base no levantamento de características de amostras selecionadas e em modelo matemático ajustado, foram simulados os desempenhos dos principais minérios das minas $\mathrm{N} 4 \mathrm{e}$ N5 de Carajás, no circuito industrial de moagem ali instalado.

Para tanto, empregou-se o método desenvolvido por um dos autores (Delboni Jr et al., 2001) para determinação da função distribuição de quebra de cinco tipos de minério de Carajás, provenientes das frentes de lavra das minas N4 e N5.

\section{Materiais e métodos}

Para obtenção de amostras destinadas a ensaios de caracterizações, foram realizadas amostragens nas frentes de lavra das minas de Carajás. Os resultados dos ensaios obtidos foram empregados para a simulação de processamento no simulador JKSimMet.

\subsection{Amostragens}

Foram coletadas amostras de ROM provenientes das frentes de lavra N4E, N4W Central, N4WN e N5E.

As amostras foram homogeneizadas por pilha alongada e, posteriormente, quarteadas. Uma alíquota foi empregada para determinar a distribuição granulométrica no Laboratório Físico de Carajás, sendo o material restante encaminhado ao Laboratório de Simulação e Controle do Departamento de Engenharia de Minas e de Petróleo da Escola Politécnica da Universidade de São Paulo (LSC/EPUSP). No LSC, as amostras foram submetidas a ensaios de caracterização tecnológica quanto à fragmentação.

\subsection{Distribuição granulométrica}

As amostras quarteadas foram submetidas ao procedimento-padrão da Vale para determinação de distribuição granulométrica. Tal procedimento inclui peneiramento a seco, para as frações mais grossas que $150 \mu \mathrm{m}$, peneiramento a úmido e o emprego de ciclosizer, para as frações mais finas que $45 \mu \mathrm{m}$.

\subsection{Drop Weight Test - DWT}

Os ensaios de DWT foram realizados segundo a metodologia do JKMRC, descrita na Parte 1 desse artigo.

\subsection{Simulação}

Os modelos matemáticos foram ajustados com base na função distribuição de quebra determinada para o minério amostrado na alimentação do circuito de moagem, conforme descrito na Parte 1 desse artigo. Tais modelos foram utilizados nos exercícios de simulação, empregando-se o simulador JKSimMet.

Foram utilizados o modelo do misturador perfeito, para os moinhos, e o modelo de curva de partição, para os ciclones.

\section{Resultados e discussão}

\subsection{Distribuição granulométrica}

As distribuições granulométricas dos minérios amostrados, nas diferentes frentes de lavra, seguem apresentadas na Tabela 1.

Entre os minérios analisados, aquele que apresentou uma distribuição granulométrica mais fina foi o N4E e a mais grossa foi o N4WN.

\subsection{Função distribuição de quebra}

Foram submetidas aos ensaios de DWT seis amostras de minério de Carajás: uma proveniente da alimentação do circuito de moagem e as demais provenientes das frentes de lavra das minas N4 e N5.

Conforme foi descrito na Parte 1 desse artigo, os ensaios foram conduzidos sobre amostras preparadas com tamanho entre 22,4 e 19,0 mm. Os resultados obtidos, os valores de A e b ajustados e de $t_{10}{ }^{*}$ seguem na Tabela 2. As respectivas funções distribuição de quebra seguem na Tabela 3.

A partir dos valores de $t_{10}{ }^{*}$ e das funções distribuição de quebra obtidas, observam-se dois tipos de minério com menor resistência ao impacto: o minério proveniente da frente de lavra N4WN e o minério amostrado na usina.

Tabela 1 - Resultados de distribuição granulométrica.

\begin{tabular}{c|c|c|c|c}
\hline \multirow{2}{*}{$\begin{array}{c}\text { Malha } \\
\text { (mm) }\end{array}$} & \multicolumn{4}{|c}{ Porcentagem passante acumulada (\%) } \\
\cline { 2 - 5 } & N4E & N4WN & N4W Central & N5E \\
\hline 50 & 100 & 100 & 100 & 100 \\
\hline 25 & 98,8 & 96,6 & 99,7 & 98,6 \\
\hline 12,5 & 94,3 & 86,7 & 94,7 & 96,4 \\
\hline 6,30 & 84,3 & 68,2 & 84,9 & 89,7 \\
\hline 1,00 & 55,9 & 35,5 & 52,7 & 48,3 \\
\hline 0,106 & 22,5 & 11,7 & 15,6 & 15,9 \\
\hline 0,007 & 5,4 & 2,9 & 4,2 & 3,8 \\
\hline
\end{tabular}


Bianca Foggiatto e Homero Delboni Júnior

\subsection{Simulação}

Empregando os modelos calibrados dos moinhos e dos ciclones, foram conduzidos exercícios de simulação para as diferentes frentes de lavra de Carajás. Dois parâmetros foram simulados:

- Distribuição granulométrica da alimentação nova.

- Função distribuição de quebra.

As variáveis operacionais, as dimensões dos equipamentos, o índice de moabilidade (WI de Bond), bem como os erros atribuídos aos dados de entrada do circuito (vazões, porcentagem de sólidos e distribuição granulométrica), foram mantidos constantes, com o intuito de isolar os efeitos da função distribuição de quebra nas simulações.

O Work Index (WI) também é um parâmetro de entrada do modelo. Contudo tal parâmetro não é empregado no ajuste de modelos, mas, sim, na etapa de simulação com uma referência de moabilidade (JKSimMet, 2003).

Para avaliar as variações de distribuição granulométrica dos produtos obtidos, foram selecionados três parâmetros: a porcentagem retida em 106 $\mu \mathrm{m}$ e as porcentagens passantes em 45 e $7 \mu \mathrm{m}$

Os valores simulados foram, portanto, comparados com o caso-base. Tais resultados são apresentados na Tabela 4.

O produto da moagem cuja distribuição granulométrica mais se aproximou daquele obtido no caso-base foi o da amostra N4WN. Os produtos que apresentaram quantidade de finos significativamente maior foram N4W Central e N4E.

Os overflows dos ciclones simulados apresentaram distribuições granulométricas simuladas mais grossas do que a do caso-base, com exceção da frente de lavra N4W Central. Essa frente se destaca pela similaridade da sua granulometria com aquela obtida no caso-base.

Também se destaca o overflow obtido na a frente de lavra N5E, cuja maior diferença foi de $1,0 \%$ e ocorreu para a fração passante em $7 \mu \mathrm{m}$.
Tabela 2 - Resultados dos ensaios de DWT.

\begin{tabular}{|c|c|c|c|c|c|}
\hline Amostra & $E c s(\mathrm{kWh} / \mathrm{t})$ & $t_{10}(\%)$ & $A$ & $b$ & $t_{10}$ * \\
\hline \multirow{3}{*}{ N4E } & 2,53 & 46,75 & \multirow{3}{*}{27,0} & \multirow{3}{*}{10,0} & \multirow{3}{*}{34,6} \\
\hline & 1,03 & 36,85 & & & \\
\hline & 0,27 & 18,04 & & & \\
\hline \multirow{3}{*}{ N4WN } & 2,51 & 37,02 & \multirow{3}{*}{40,0} & \multirow{3}{*}{9,0} & \multirow{3}{*}{46,9} \\
\hline & 1,01 & 25,74 & & & \\
\hline & 0,25 & 13,69 & & & \\
\hline \multirow{3}{*}{ N4W Central } & 2,54 & 49,45 & \multirow{3}{*}{27,5} & \multirow{3}{*}{11,9} & \multirow{3}{*}{36,6} \\
\hline & 1,05 & 38,61 & & & \\
\hline & 0,28 & 29,31 & & & \\
\hline \multirow{3}{*}{ N5E } & 2,54 & 36,81 & \multirow{3}{*}{30,3} & \multirow{3}{*}{10,7} & \multirow{3}{*}{38,4} \\
\hline & 1,03 & 24,68 & & & \\
\hline & 0,28 & 10,33 & & & \\
\hline \multirow{3}{*}{ AL MO } & 2,53 & 46,75 & \multirow{3}{*}{35,6} & \multirow{3}{*}{15,1} & \multirow{3}{*}{47,1} \\
\hline & 1,03 & 36,85 & & & \\
\hline & 0,27 & 18,04 & & & \\
\hline
\end{tabular}

Tabela 3 - Funções distribuição de quebra.

\begin{tabular}{c|c|c|c|c|c}
\hline & \multicolumn{5}{|c}{ Porcentagem retida simples (\%) } \\
\cline { 2 - 6 } & AL MO & N4E & N4WN & N4W Central & N5E \\
\hline $\boldsymbol{t}_{\boldsymbol{1 0}}$ & 0 & 0 & 0 & 0 & 0 \\
\hline $\boldsymbol{t}_{\boldsymbol{2}}$ & 0 & 6 & 0 & 4 & 2 \\
\hline $\boldsymbol{t}_{\boldsymbol{4}}$ & 19 & 26 & 19 & 25 & 24 \\
\hline $\boldsymbol{t}_{\boldsymbol{1 0}}$ & 30 & 31 & 30 & 32 & 33 \\
\hline $\boldsymbol{t}_{\mathbf{2 5}}$ & 24 & 17 & 23 & 18 & 19 \\
\hline $\boldsymbol{t}_{\mathbf{5 0}}$ & 11 & 8 & 11 & 8 & 8 \\
\hline $\boldsymbol{t}_{\mathbf{7 5}}$ & 5 & 4 & 5 & 4 & 4 \\
\hline $\boldsymbol{f i n o s}$ & 11 & 8 & 11 & 9 & 9 \\
\hline
\end{tabular}

A partir das porcentagens de sólidos simuladas, também foram calculadas as cargas circulantes, as quais são apresentadas na Tabela 5.

Nota-se que, para N4E e N4W Central, a carga circulante foi, significativamente, menor, para a mesma vazão de alimentação nova. Analogamente a frente N4WN apresentou maior carga circulante relativa. 
Influência da função distribuição de quebra na modelagem e simulação do circuito de moagem de Carajás...

Nota-se, portanto, que há necessidade de ajustes relativamente amplos na carga circulante do circuito, conforme os diferentes tipos de minério processados no circuito de moagem de Carajás. A estratégia de manter-se a carga circulante constante é, portanto, inadequada, conforme demonstraram os resultados das simulações.

Se convenientemente ajustado, o circuito de moagem de Carajás deve processar minérios N4WN e N5E e gerar produtos com granulometria praticamente idênticas àquelas verificadas no caso base. Esperam-se, portanto, recuperações em massa semelhantes ao caso-base na etapa de deslamagem.

Já minérios como N4E e N4W Central indicam produtos com excesso de finos, o que resultaria em decréscimo na recuperação em massa global do circuito de moagem e deslamagem de Carajás.

\section{Comentários finais}

Os exercícios de simulação resultaram em um recurso muito útil para prever o desempenho do circuito industrial e permitiram avaliar a influência da função distribuição de quebra. A análise comparativa dos produtos simulados, bem como dos demais parâmetros do circuito, mostrou diferenças significativas entre as frentes de lavra.

As simulações conduzidas apontam para potenciais de processamento dos minérios cujo comportamento na moagem era até então desconhecido.

\section{Agradecimentos}

À FAPESP, pelo apoio técnico e financeiro. À Vale, pelo apoio técnico e financeiro, em especial a Marco Túlio Santiago, José Antonio Oliveira, Francisco Patto, Vânia Oliveira, Eduardo Veríssimo, Ercílio Almeida, Rogério Patente e à equipe do Laboratório Físico de Carajás. Aos funcionários do Laboratório de Simulação e Controle da Universidade de São Paulo, pelo apoio nos trabalhos experimentais.
Tabela 4 - Parâmetros simulados do produto da moagem e do overflow dos ciclones.

\begin{tabular}{|c|c|c|c|c|c|}
\hline \multirow[t]{2}{*}{ Cenário } & \multirow{2}{*}{$\begin{array}{c}\text { Tamanho } \\
(\mu \mathrm{m})\end{array}$} & \multicolumn{2}{|c|}{$\begin{array}{c}\text { Passante } \\
\text { acumulado (\%) }\end{array}$} & \multicolumn{2}{|c|}{ Diferença (\%) } \\
\hline & & PROD. & $\mathrm{O} / \mathrm{F}$ & PROD. & $\mathrm{O} / \mathrm{F}$ \\
\hline \multirow{3}{*}{$\begin{array}{l}\text { Caso- } \\
\text { Base }\end{array}$} & 106 & 43,3 & 99,1 & & \\
\hline & 45 & 22,4 & 83,4 & & \\
\hline & 7 & 9,3 & 37,4 & & \\
\hline \multirow{3}{*}{ N4E } & 106 & 22,5 & 99,6 & $-20,8$ & $+0,5$ \\
\hline & 45 & 16,1 & 90,2 & $-6,3$ & $+6,8$ \\
\hline & 7 & 5,4 & 53,3 & $-3,9$ & $+15,9$ \\
\hline \multirow{3}{*}{ N4WN } & 106 & 42,2 & 99,1 & $-1,1$ & 0 \\
\hline & 45 & 21,4 & 83,0 & -1 & $-0,4$ \\
\hline & 7 & 8,8 & 37,0 & $-0,5$ & $-0,4$ \\
\hline \multirow{3}{*}{$\begin{array}{l}\text { N4W } \\
\text { Central }\end{array}$} & 106 & 15,6 & 99,7 & $-27,7$ & $+0,6$ \\
\hline & 45 & 11,7 & 90,2 & $-10,7$ & $+6,8$ \\
\hline & 7 & 4,2 & 53,5 & $-5,1$ & $+16,1$ \\
\hline \multirow{3}{*}{ N5E } & 106 & 45,4 & 99,2 & $+2,1$ & $+0,1$ \\
\hline & 45 & 23,7 & 83,8 & $+1,3$ & $+0,4$ \\
\hline & 7 & 10,1 & 38,4 & $+0,8$ & $+1,0$ \\
\hline
\end{tabular}

Tabela 5 - Parâmetros simulados do produto da moagem e do overflow dos ciclones.

\begin{tabular}{c|c|c|c|c}
\hline \multirow{2}{*}{ Cenário } & \multicolumn{3}{|c|}{ Porcentagem de sólidos } & \multirow{2}{*}{$\begin{array}{c}\text { Carga circulante } \\
\text { (\%) }\end{array}$} \\
\cline { 2 - 4 } & Alim. & O/F & U/F & 426 \\
\hline Caso-Base & 55,5 & 23,7 & 81,0 & 233 \\
\hline N4E & 43,8 & 23,4 & 69,7 & 449 \\
\hline N4WN & 56,5 & 23,7 & 81,8 & 218 \\
\hline N4W Central & 42,6 & 23,4 & 68,2 & 399 \\
\hline N5E & 54,2 & 23,7 & 80,0 & \\
\hline
\end{tabular}

\section{Referências bibliográficas}

DELBONI JUNIOR, H., CHIEREGATI, A. C. Nova metodologia de caracterização de minérios aplicada a projetos de moinhos AG/SAG. In: ENCONTRO NACIONAL DE TRATAMENTO DE MINÉRIOS E METALURGIA EXTRATIVA, 17. Anais... Rio de Janeiro: CETEM/MCT, 2001. v. 3. p. 49-54.

JULIUS KRUTTSCHNITT TECH LTD. The JKSimMet steady state mineral processing simulator: full manual. Version 5.1. Indooroopilly, Qld, 2003. p. A69-A80.

Artigo recebido em 26/12/2008 e aprovado em 27/10/2009. 